\title{
A Novel Approach to Automatic Color Matching
}

\author{
Cornelio Yáñez, Edgardo Felipe-Riveron, I. López-Yáñez, and R. Flores-Carapia \\ Center for Computing Research, National Polytechnic Institute, Mexico, \\ Juan de Dios Batiz s/n, Col. Nueva Industrial Vallejo, C.P. 07738
}

\begin{abstract}
In this paper the design and operation of an Automatic Color Matching system is presented. This novel system takes advantage of the improvements introduced by Alpha-Beta associative memories, an efficient, unconventional model of associative memory of recent creation. The results are demonstrated through experiments on a relatively small database with 1001 samples prepared by the authors. However, the approach is considered valid according to the tendency of the results obtained, in part, thanks to the performance exhibited by Alpha-Beta associative memories.
\end{abstract}

Keywords: Color matching, Autoassociative memory, Alpha-Beta associative memories.

\section{Introduction}

Color mapping or color matching is a process by mean of which, a color is transformed to its visually closest color in a given destination color space. The final goal of the system herein presented consisted of industrial application, which is related to the problems faced by companies that manufacture paints. It is well known that usually, the factories that produce paints match colors through a color swatchbook prepared from the paints and mixes of colors particularly produced by them. The cost in equipments of color matching in real time is relatively high, and it is done only in situ, never at distance over the Internet, which is a potential future extension of our system, currently in development [1]. Many industrial, e.g. plastics, paint, and textile applications exploit color matching and mixing devices and techniques, but industries solve the problem of color matching always in a pragmatic way. Due to industrial secret there is not any information in the open literature in order to know the method the industry uses for matching colors.

On the other hand, associative memories have been an active area for research in computer sciences by roughly half a century. In this respect, computer scientists are interested in developing mathematical models that are able to learn and recall patterns (the two phases of an associative memory model) representing objects, organisms, or concepts [2-4]. The ultimate goal of an associative memory is to correctly recall complete patterns from input patterns. These patterns might be altered with noise. The classical era in associative memories is represented by Hopfield's memory [5], while in recent years the morphological associative memories [6] gave place to the rise of the Alpha-Beta $(\alpha \beta)$ associative memories [7]. This new model is based on two new 
operators (alpha and beta), and has become the most efficient and robust model of an associative memory.

In this paper we present the design and operation of an Automatic Color Matching system. This novel system takes advantage of the improvements introduced by $\alpha \beta$ associative memories. The results are demonstrated in a relatively small database with 1001 samples prepared by the authors. However, we consider valid the approach according to the tendency of the results obtained, in part, thanks to the performance exhibited by $\alpha \beta$ associative memories. The task of color matching is not a trivial one, because it depends on the preparation of color samples and their maintenance. Color matching is a process by means of which an unknown mix of color is located in a particular position in a given known color space. The system was created when it was necessary to find a procedure to achieve a fast, robust, and error-free procedure for color matching. A different possible solution to carry out the color matching task could be the Self-Organizing Maps or Kohonen Maps. However, they present some drawback which must be solved first. One major problem with SOMs is getting the right data. Another problem is that every SOM is different and finds different similarities among the sample vectors. The final major problem with SOMs is that they are very computationally expensive which is a major drawback since as the dimensions of the data increases, dimension reduction visualization techniques become more important, but unfortunately the time to compute them also increases [8].

Our solution is another alternative that gives valid results in a short time, it is not computational extensive, and does not need any previous selection or ordering of data in the data base.

The remaining of the paper is organized as follows. In Section 2 some concepts about color are provided. Section 3 is focused on explaining the $\alpha \beta$ associative memory model. Section 4 contains the core proposal and section 5 the experimental results. Section 6 is finally devoted to conclusions and directions for future research in this topic.

\section{Color}

Color is a sensation, such as warmth or a touch in our skin: it is caused by a physical reality. Color is not a property of the things that cause the sensation: grass is not green and the sky is not blue. Rather, they have physical properties that make us perceive them as green and blue, but even that is true only in some circumstances. Also, colors are not qualified in the same way by everybody that sees them. It is our trained visual system that decides on a color.

Color mixes can be obtained by means of an additive method or of a subtractive method. Primary colors in the additive method are red, green and blue. Every mix of two of them produces cyan, magenta or yellow colors. These colors are called secondary colors for this method. The mix of all of them produces white that contains all colors. Primary colors in the subtractive method are cyan, magenta and yellow. Every mix of two of them produces red, green or blue colors. These colors are called secondary colors for this method. The mix of all of them produces black that is absence of color. In both types of mix, complementary colors are those that combining together 
gives us the color resulting from the mix of three primaries of the respective method, that is, white in the additive method and black in the subtractive method. Then, complementary colors are those that when one of them is pure it means the other one is not present, so one color in pure state excludes the other. Green and magenta, red and cyan, and yellow and blue are complementary colors. In a given color space, each color has its particular unique position.

\section{$3 \alpha \beta$ Associative Memories}

Basic concepts, results and notation about associative memories were taken from the Yáñez-Márquez's PhD Thesis [8]. An associative memory $\mathrm{M}$ is a system that relates input patterns, and outputs patterns, as follows: $x \rightarrow \mathrm{M} \rightarrow y$ with $x$ and $y$ the input and output pattern vectors, respectively. Each input vector forms an association with a corresponding output vector. For $k$ integer and positive, the corresponding association will be denoted as $\left(x^{k}, y^{k}\right)$. Associative memory $\mathrm{M}$ is represented by a matrix whose $i j$-th component is $m_{i j}$. Memory $\mathrm{M}$ is generated from an a priori finite set of known associations, known as the fundamental set of associations. If $\mu$ is an index, the fundamental set is represented as: $\left\{\left(x^{\mu}, y^{\mu}\right) \mid \mu=1,2, \ldots, p\right\}$ with $p$ the cardinality of the set. If it holds that $x^{\mu}=y^{\mu}, \forall \mu \in\{1,2, \ldots, p\}, \mathrm{M}$ is autoassociative, otherwise it is heteroassociative. In this case it holds that $\exists \mu \in\{1,2, \ldots, p\}$ for which $x^{\mu} \neq y^{\mu}$.

The $\alpha \beta$ associative memories are of two kinds and are able to operate in two different modes. Operator $\alpha$ is useful at the learning phase, and operator $\beta$ is the basis for the pattern recall phase.

The core of the mathematical tools used in the $\alpha \beta$ model, are two binary operators designed specifically for these memories. These operators are defined in [9] as follows: First, we define the sets $A=\{0,1\}$ and $B=\{00,01,10\}$, then the operators $\alpha$ and $\beta$ are defined in tabular form, as shown in Table 1 .

Table 1. Definition of the Alpha and Beta operators

$\alpha: A \times A \rightarrow B$
\begin{tabular}{|c|c|c|}
\hline$x$ & $y$ & $\alpha(x, y)$ \\
\hline 0 & 0 & 01 \\
\hline 0 & 1 & 00 \\
\hline 1 & 0 & 10 \\
\hline 1 & 1 & 01 \\
\hline
\end{tabular}

\begin{tabular}{|c|c|c|}
\multicolumn{1}{c}{$\beta: B \times A \rightarrow A$} \\
\hline$x$ & $y$ & $\beta(x, y)$ \\
\hline 00 & 0 & 0 \\
\hline 00 & 1 & 0 \\
\hline 01 & 0 & 0 \\
\hline 01 & 1 & 1 \\
\hline 10 & 0 & 1 \\
\hline 10 & 1 & 1 \\
\hline
\end{tabular}

The sets $A$ and $B$, the $\alpha$ and $\beta$ operators, along with the usual $\wedge$ (minimum) y $\vee$ (maximum) operators, form the algebraic system $(A, B, \alpha, \beta, \wedge, \vee)$ which is the mathematical basis for the $\alpha \beta$ associative memories. The $i j$-th entry of the matrix $y \oplus x^{t}$ is: $\left[y \oplus x^{t}\right]_{i j}=\alpha\left(y_{i}, x_{j}\right)$. If we consider the fundamental set of patterns: 


$$
\begin{aligned}
& \left\{\left(x^{\mu}, y^{\mu}\right) \mid \mu=1,2, \ldots, p\right\} \text { where } x^{\mu} \in A^{n} \text { and } y^{\mu} \in A^{m} \text { then: } \\
& \left\lfloor y^{\mu} \oplus\left(x^{\mu}\right)^{t}\right\rfloor_{i j}=\alpha\left(y_{i}^{\mu}, x_{j}^{\mu}\right) .
\end{aligned}
$$

Because there are two kinds of $\alpha \beta$ associative memories $\vee$ and $\Lambda$, and considering that each of these kinds is able to operate in two different modes, heteroassociative and autoassociative, we have four different available choices. In this issue, we only talk about the $\alpha \beta$ autoassociative memories of kind $V$. Therefore, the fundamental set takes the form: $\left\{\left(x^{\mu}, x^{\mu}\right) \mid \mu=1,2, \ldots, p\right\}$. Besides, the input and output patterns have the same dimension $n$, and the memory is a square matrix: $\mathrm{V}=\left\lfloor v_{i j}\right\rfloor_{n \times n}$.

\section{Learning Phase (two steps)}

\section{STEP 1:}

For each $\mu=1,2, \ldots, p$, and from $\left(x^{\mu}, x^{\mu}\right)$ build the matrix: $\left[x^{\mu} \oplus\left(x^{\mu}\right)^{t}\right]_{n \times n}$.

\section{STEP 2:}

Apply the binary $\vee$ operator to the matrices obtained in step 1 to get $\mathbf{V}$ as follows: $\mathbf{V}=\bigvee_{\mu=1}^{p}\left[x^{\mu} \oplus\left(x^{\mu}\right)^{t}\right]$. The $i j$-th entry is given as: $v_{i j}=\bigvee_{\mu=1}^{p} \alpha\left(x_{i}^{\mu}, x_{j}^{\mu}\right)$. It is obvious that, $v_{i j} \in B, \forall i \in\{1,2, \ldots, n\}, \forall j \in\{1,2, \ldots, n\}$.

\section{Recalling Phase}

A pattern $x^{\Phi}$, with $\omega \in\{1,2, \ldots, p\}$ is presented to the $\alpha \beta$ autoassociative memory of kind $\mathbf{V}$ and the following operation is done: $\mathrm{V} \bigcap_{\beta} x^{\varpi}$. The result is a column vector of dimension $n$, where: $\left(\mathbf{V} \bigcap_{\beta} x^{\varpi}\right)_{i}=\bigwedge_{j=1}^{n} \beta\left\{\left[\bigvee_{j=1}^{n} \alpha\left(x_{i}^{\mu}, x_{j}^{\mu}\right)\right], x_{j}^{\varpi}\right\}$.

The greatest limitation of $\alpha \beta$ associative memories is perhaps that they only work with binary data, being unable to manipulate integer or real numbers. However, there have been numerous efforts to extend the original model [10-11]. With these advances, $\alpha \beta$ memories can be applied to binary and integer pattern recognition.

\section{Our Proposal}

In this section the proposal of the intelligent system for Automatic Color Matching is presented. First, it will be explained how the color samples database was prepared, and the methodology used to take measurements. Based on these and the concepts given on sections 2 and 3, the design and operation of the proposed intelligent system is discussed. 


\subsection{Preparation of Color Samples}

If color samples to be measured are not rigorously prepared, the color recognition is prone to errors, making this preparation of utmost importance. The color samples produced by mixing three primary colors cyan, magenta and yellow, together with black and distilled water in previously defined proportions will constitute the patterns to be recognized. On the other hand, colors parameters can be measured by colorimeters, spectrophotometers, and other equipments. In our case a spectrophotometer was used. The color measurement by means of a spectrophotometer could be by transmittance or by reflectance. Thus, several considerations were imposed to the color samples to be used to carry out our experiments.

First, the type of the color source must be selected; that is, the material having the required primary colors to prepare the mixes to be recognized. It could be liquid, powder, pastes, and others. If transmittance is used to measure the colors, like in the case of liquids, the use of expensive quartz vessels is obligatory, since the transparence of the vessel must be considerably high in order to guarantee that its contribution in color be practically null. Therefore it was decided to measure colors by reflectance. For all these reasons, it was decided to use as the color source to prepare the color samples the Hewlett Packard cyan, magenta, yellow and black printer inks. It was used also distilled water in order to simulate the presence of the white color when the mixes were prepared over a white substrate [12].

As a second step, the choice of the substrate where the printer inks will be applied became of great importance. The main characteristic to be fulfilled by the substrate was that its contribution in color when the inks are applied over it, be a minimum and non-dependent on the mix prepared, according to our convenience. A second requirement was that the application of a liquid or a paste onto it does not moisten the substrate in such a form that makes it useless. Also its white color and its reflectivity must be uniform in the whole range of visible wavelengths. With all this in mind, three types of substrate were considered: photographic paper, white couche paper and common white kromekote paper. The reflectance vs wavelength curves obtained from the spectrophotometer for these three types of papers showed that the kromekote paper gives a better behavior than the other two.

The number of drops of ink to be deposited over the substrate was crucial also. The first condition to be fulfilled was that the reflectance of the paper does not affect the reflectance of the color sample. The second condition is that an excessive number of drops do not moisten the paper, in a measure that the color reflectances vary with the characteristics of the paper. In this sense some tests were done. Table 2 show the luminance level $\mathrm{L}^{*}$ of the three primary colors cyan, magenta and yellow, respectively, when measured for 1 to 10 ink drops, with respect to the standard cyan, magenta and yellow colors reflectance given by the calibrated spectrophotometer.

It may be noted that except for yellow, for every color the luminance with six drops of ink on the substrate is similar to the standard one (first row in the tables), which means that the "pure" primary color covers completely the substrate; that is, the influence of the higher luminance of the white color of the substrate is minimum or nearly null. It can be noted also that yellow gives higher luminance values than the other two primary colors, shows a more uniform characteristic and its luminance value is nearest to that of the substrate (94.29). The fact that only five drops of yellow 
cover the substrate (lesser luminance value), it means that it is the color that better covers the substrate. The variable values of luminance obtained in all cases when more than six drops of ink were used, is a sign that the substrate has been already moistened and due to this, the color properties changed. Then, as a conclusion, using only six drops of mixed inks carried out the covering of the substrate with all color samples.

Table 2. Luminance values for cyan, magenta and yellow ink

\begin{tabular}{|c|c|c|c|c|c|c|}
\hline $\begin{array}{c}\text { Color of } \\
\text { Ink }\end{array}$ & \multicolumn{2}{|c|}{ Cyan ink } & \multicolumn{2}{c|}{ Magenta ink } & \multicolumn{2}{c|}{ Yellow ink } \\
\hline $\begin{array}{c}\text { Number of } \\
\text { drops }\end{array}$ & $\begin{array}{c}\text { Luminance } \\
\text { Level }\end{array}$ & Difference & $\begin{array}{c}\text { Luminance } \\
\text { Level }\end{array}$ & Difference & $\begin{array}{c}\text { Luminance } \\
\text { Level }\end{array}$ & Difference \\
\hline Standard & 59.05 & -35.90 & 49.59 & -44.28 & 87.39 & -6.65 \\
\hline 1 & 69.08 & 10.02 & 60.94 & 11.35 & 87.69 & 0.30 \\
\hline 2 & 66.83 & 7.78 & 57.01 & 7.42 & 87.44 & 0.05 \\
\hline 3 & 67.80 & 8.75 & 55.07 & 5.48 & 87.42 & 0.03 \\
\hline 4 & 66.86 & 7.81 & 53.81 & 4.22 & 87.37 & -0.01 \\
\hline 5 & 60.59 & 1.53 & 52.96 & 3.36 & 87.12 & -0.27 \\
\hline 6 & 58.39 & 0.66 & 50.01 & -0.42 & 87.65 & -0.26 \\
\hline 7 & 62.34 & 3.29 & 53.43 & 3.84 & 85.18 & $-2-21$ \\
\hline 8 & 60.17 & 1.12 & 50.49 & 0.90 & 85.53 & -1.86 \\
\hline 9 & 60.69 & 1.64 & 52.98 & 3.38 & 84.92 & -2.47 \\
\hline 10 & 61.37 & 2.32 & 48.35 & -1.25 & 87.78 & 0.39 \\
\hline
\end{tabular}

To achieve different shadows and tints of pure primary colors and their mixes, it was necessary to mix pure primary color inks with black ink and distilled water in the corresponding proportions, respectively. The white color of the substrate contributed to give tint when distilled water was used in the mixes. Afterward, other particular mixes were prepared using the corresponding shadowed and tinted primaries. The total number of drops used in the preparation of every color mix was limited always to one hundred drops. Each color mix prepared was kept in a small previously sterilized dropper glass, sealed with a cap and protected from light in order to guarantee that its quality does not deteriorate.

To calculate the exact number of possible color patterns that must be prepared according to the conditions stated, generating function models were used [13]. Suppose $a_{r}$ is the number of ways to select $r$ objects in a certain procedure. Then $g(x)$ is a generating function for $a_{r}$ if $g(x)$ has the polynomial expansion:

$$
g(x)=a_{0}+a_{1} x+a_{2} x^{2}+\cdots+a_{r} x^{r}+\cdots+a_{n} x^{n} .
$$

For example as: $(1+x)^{n}=1+\left(\begin{array}{l}n \\ 1\end{array}\right) x+\left(\begin{array}{l}n \\ 2\end{array}\right) x^{2}+\cdots+\left(\begin{array}{l}n \\ r\end{array}\right) x^{r}+\cdots+\left(\begin{array}{l}n \\ n\end{array}\right) x^{n}$. Then $g(x)=(1+x)^{n}$ is the generating function for $a_{r}=C(n, r)$, the number of ways to select an $r$-subset from an $n$-set. The problem of determining the coefficient of $x^{r}$ when we multiply several such polynomial factors together can be restated in 
terms of exponents. As an example, consider the expansion of $\left(1+x+x^{2}\right)^{4}$. The number of formal products $x^{e_{1}} x^{e_{2}} x^{e_{3}} x^{e_{4}}, 0 \leq e_{i} \leq 2$ equaling $x^{r}$ in the expansion, will be the number of integer solutions to: $e_{1}+e_{2}+e_{3}+e_{4}=r, 0 \leq e_{i} \leq 2$. In our case, the total number of drops of the five possible components to create each color mix was limited to 100 drops for convenience. Additionally, the concentration of samples created for each one out of five components was established in steps of ten drops, that is, $r=100 / 10=10$ steps. This means that: $e_{1}+e_{2}+\cdots+e_{5}=10$, where $0 \leq e_{i} \leq 10$. This is equivalent to solving the polynomial: $P(x)=\left(x^{0}+x^{1}+\cdots+x^{9}+x^{10}\right)^{5}$. In the expansion of this polynomial $P(x)$ in its formal products, the coefficient $a_{10}$ of $x^{10}$ gives the total number of combinations under our conditions. That coefficient is 1001 . Then, the number of combinations (to be) prepared was 1001 combinations.

\subsection{Measurements}

In taking measurements, the spectrophotometer was calibrated to measure the samples under a known specific set of conditions. These conditions involve the illuminant (light source) type, the observer's viewing angle, and the spectral exclusion (not taking gloss into consideration). The spectrophotometer used was a Milton Roy COLOR MATE $^{\mathrm{TM}}$ HDS Color Analyzer. Before its use and after warmed, it was always meticulously calibrated with a white tile accompanying the equipment. The parameters established in the equipment by us to measure the color samples were: Observation Angle: $10^{\circ}$; Sight Window (Area of View): small; Color Space: CIE L ${ }^{*}{ }^{*} b^{*}$; Illuminants: D65 and D50; and Average number of lectures: 5.

Five lectures were done facing onto it different zones of the covered substrate. The equipment then averaged the results of the five lectures and finally gave us thirty one values of the reflectance of the sample for each one of the thirty one wavelengths ranging from 400 to 700 nanometers of the visible spectrum, in steps of ten, of the standard and the color sample, as well as the difference between both values. The standard color could be selected by the user, between the standard white, standard black, one of the primary color selected from cyan, magenta and yellow, or another one of our convenience. From the two illuminants selected, that is D50 and D65, the spectrophotometer gave us the values of the components $\mathrm{L}^{*}, \mathrm{a}^{*}, \mathrm{~b}^{*}$ of the color model CIELAB selected and the values of the three primary three-stimulus values $\mathrm{X}, \mathrm{Y}$ and $\mathrm{Z}$, for both the standard and the sample, together with the respective difference. In order to carry out the color matching, the value of $\mathrm{L}^{*}$ related to the illuminant D65 was used as reference for daylight. Then, after reading the 1001 color samples, prepared with colors cyan, magenta, yellow, black and distilled water in steps of ten drops, and taking into account that the total number of drops be 100 drops for each mix prepared and used 6 drops to cover the substrate, a database was created with patterns of thirty one values of the reflectance, which vary from 6.24 to 102.58 .

\subsection{System Description}

The system consists of three modules: the input module, the processing module and the output module; and operates in two phases: the learning phase and the pattern 
recall phase. The first module has as main purpose to convert each input pattern made up of 31 real values into its corresponding binary pattern, requirement imposed for the design and operation of the $\alpha \beta$ associative memories. The third module does the reverse procedure: converts the binary patterns which result from operating the system into their corresponding 31 real components patterns, which make up the pattern for a specific color. Module 2 is the core of the system, and is conformed by two complementing redundant sub-modules: the first sub-module is an autoassociative $\alpha \beta$ memory of type max, and the second sub-module is an autoassociative $\alpha \beta$ memory of type min. In this intelligent system, the fundamental patterns of each $\alpha \beta$ associative memory are precisely the patterns of 31 real valued reflectance components representing the different colors. Since the memories are autoassociative, it holds that $x^{\mu}=y^{\mu}, \forall \mu \in\{1,2, \ldots, p\}$, where $p$ is the fundamental set cardinality; that is, $p=1001$.

Learning phase.-

- Every pattern in the fundamental set is presented to the system and module 1 converts into binary patterns the 31 real component patterns.

- Both $\alpha \beta$ associative memories, max and min, do their respective learning phases.

Matching Phase.-

- An unknown 31 real valued pattern is presented to the system and module 1 converts it to a binary pattern.

- Each $\alpha \beta$ associative memory, max and min, executes its pattern recall phase, producing two binary patterns.

- Module 3 converts each of these two binary patterns into a 31 real valued pattern.

Given the redundancy of both submodules of module 2, it is expected that both obtained patterns be equal. However, they were different. The worst case occurs when both patterns are a valid version of a color resulting from the matching. It is left to the user to select by visual inspection the best of them.

\section{Experimental Results}

The experimental design is quite simple. In each individual experiment, the following was done: (a) At random one out of 1001 samples was selected from the database. Then, the database had only 1000 elements (color combination) in this case; (b) the learning phase of the system was undertaken with the resulting 1000 patterns database; (c) the color that was retired in step 1 was used as sample. Then the results given by the system was analyzed by several human observers, who dictated whether the color resulting from the matching was close enough to the testing color, or not.

In Table 3 are shown the values of the number of drops of 24 samples to match selected at random from the database, the number of drops of the matching calculated by the matching module and the majority opinion of the observers group.

In this table we can notice that all results are positive, except when pure colors were presented to the system, that is, colors which have presence of only one primary color. Since these colors are known in advance, the system failure with them is irrelevant. 
Table 3. Experimental results

\begin{tabular}{|c|c|c|c|c|c|c|c|c|c|c|}
\hline \multicolumn{5}{|c|}{$\begin{array}{l}\text { Number of drops of the color sample to } \\
\text { match }\end{array}$} & \multicolumn{5}{|c|}{$\begin{array}{l}\text { Number of drops of the matching with the } \\
\text { color sample }\end{array}$} & \multirow{2}{*}{$\begin{array}{l}\text { Yes/ } \\
\text { No }\end{array}$} \\
\hline Cyan & Mag. & Yellow & Water & Black & Cyan & Mag. & Yellow & Water & Black & \\
\hline 0 & 60 & 20 & 0 & 20 & 0 & 40 & 20 & 20 & 20 & yes \\
\hline 80 & 0 & 0 & 0 & 20 & 0 & 0 & 0 & 0 & 100 & no \\
\hline 40 & 0 & 30 & 10 & 20 & 50 & 0 & 30 & 0 & 20 & yes \\
\hline 50 & 30 & 10 & 0 & 10 & 40 & 30 & 10 & 10 & 10 & yes \\
\hline 40 & 30 & 20 & 0 & 10 & 40 & 30 & 10 & 10 & 10 & yes \\
\hline 20 & 0 & 50 & 30 & 0 & 20 & 0 & 60 & 20 & 0 & yes \\
\hline 30 & 10 & 0 & 10 & 50 & 20 & 10 & 20 & 20 & 30 & yes \\
\hline 0 & 10 & 0 & 10 & 80 & 0 & 10 & 0 & 0 & 90 & yes \\
\hline 0 & 40 & 0 & 0 & 60 & 20 & 10 & 10 & 0 & 60 & no \\
\hline 0 & 50 & 40 & 10 & 0 & 0 & 60 & 30 & 10 & 0 & yes \\
\hline 30 & 50 & 0 & 0 & 20 & 30 & 40 & 0 & 10 & 20 & yes \\
\hline 0 & 20 & 60 & 20 & 0 & 0 & 40 & 60 & 0 & 0 & yes \\
\hline 20 & 10 & 10 & 50 & 10 & 30 & 10 & 10 & 30 & 20 & yes \\
\hline 0 & 0 & 70 & 30 & 0 & 0 & 0 & 40 & 60 & 0 & no \\
\hline 0 & 0 & 10 & 80 & 10 & 0 & 0 & 10 & 70 & 20 & yes \\
\hline 0 & 50 & 10 & 30 & 10 & 0 & 50 & 0 & 30 & 20 & yes \\
\hline 0 & 40 & 0 & 0 & 60 & 20 & 10 & 10 & 0 & 60 & no \\
\hline 30 & 0 & 0 & 60 & 10 & 40 & 0 & 0 & 40 & 20 & no \\
\hline 40 & 0 & 50 & 0 & 10 & 40 & 0 & 30 & 20 & 10 & yes \\
\hline 20 & 0 & 10 & 50 & 20 & 30 & 0 & 10 & 20 & 40 & yes \\
\hline 0 & 60 & 30 & 10 & 0 & 0 & 70 & 30 & 0 & 0 & yes \\
\hline 0 & 10 & 20 & 0 & 70 & 0 & 10 & 10 & 20 & 60 & yes \\
\hline 0 & 20 & 20 & 50 & 10 & 0 & 30 & 20 & 30 & 20 & yes \\
\hline 10 & 60 & 30 & 0 & 0 & 10 & 50 & 30 & 10 & 0 & yes \\
\hline
\end{tabular}

\section{Conclusions}

This work has been oriented toward the use of $\alpha \beta$ associative memories for colors matching. The universe of samples is a relatively small database with 1001 carefully prepared samples. The experiments consisted in excluding a randomly selected sample from the full database, then building both $\alpha \beta$ memories with the remaining 1000 samples, in order to operate the system with the excluded pattern. After the system delivered the recalled patterns, a group of observers decided whether the results were satisfactory or not. The results manifest the validity of its use in a relatively easy way, in this relatively particular application, except when presented with pure colors; that is, colors which have presence of only one primary color. Since these colors are known in advance, the system failure with them is irrelevant. Therefore, the approach can be considered valid according to the tendency of the results obtained. 


\section{Acknowledgements}

The authors would like to thank the Instituto Politécnico Nacional (Secretaría Académica, COFAA, SIP, and CIC), the CONACyT, and SNI for their economical support to develop this work.

\section{References}

1. Aquino, D., Cervantes, F., Linares, B., Felipe, E., De la Cruz, C.: Telecolorimetría basada en el reconocimiento de patrones de color, Thesis. Interdisciplinary Professional School in Engineering and Advanced Technologies, National Polytechnic Institute, Mexico (2004)

2. T. Kohonen.: Correlation Matrix Memories. IEEE Transactions on Computers. 21(4). (1972) 353-359

3. Kohonen, T.: Self-Organization and Associative Memory. Springer-Verlag, Berlin Heidelberg New York (1989)

4. Hassoun, M. H.: Associative Neural Memories. Oxford University Press, New York (1993)

5. Hopfield, J. J.: Neural networks and physical systems with emergent collective computational abilities, Proceedings of the National Academy of Sciences, 79 (1982), 2554 2558

6. Ritter, G. X., Sussner, P., Diaz-de-Leon, J. L.: Morphological Associative Memories. IEEE Transactions on Neural Networks. 9 (1998) 281-293

7. Yáñez-Márquez, C., Díaz de León-Santiago, J. L.: Memorias Asociativas Basadas en Relaciones de Orden y Operaciones Binarias. Computación y Sistemas. México, 6(4). (2003) 300-311

8. Honkela, T.: Self-Organizing Maps in Natural language Processing, PhD Thesis (1997)

9. Yáñez-Márquez, C.: Associative Memories Based on Order Relations and Binary Operators (In Spanish). PhD Thesis. Center for Computing Research, México (2002)

10. Yáñez-Márquez, C., Díaz de León-Santiago, J. L., Salgado, J. C.: New V-Alpha-Beta Associative Memories able to Learn and Recall Patterns with Integer Components. Technical Report 210, Blue Series. National Polytechnic Institute. Center for Computing Research, México (2005)

11. Sossa, H., Barrón, R., Cuevas, F., Aguilar, C.: Associative Gray Level Pattern Processing Using Binary Decomposition and $\alpha \beta$ Memories. Neural Processing Letters, Vol. 22 (1) (2005) 85

12. Zhang, H., Dong Bi, J., Back, B.: The Design and Implementation of Color Matching System Based on Back Propagation, Lecture Notes in Computer Science, Vol. 2358, SpringerVerlag, Berlin (2002) 557-566

13. Tucker A.: Applied Combinatorics, John Wiley \& Sons, $4^{\text {th }}$ Edition, Chapter 6 (2002) 